\title{
Gene expression profiling via bioinformatics analysis reveals biomarkers in laryngeal squamous cell carcinoma
}

\author{
GUO-FANG GUAN $^{1}$, YING ZHENG ${ }^{2}$, LIAN-JI WEN ${ }^{1}$, DE-JUN ZHANG ${ }^{1}$, \\ DUO-JIAO YU ${ }^{1}$, YAN-QING LU ${ }^{1}$, YAN ZHAO ${ }^{1}$ and $\mathrm{HUI} \mathrm{ZHANG}^{1}$ \\ ${ }^{1}$ Department of Otolaryngology, Head and Neck Surgery, The Second Hospital of Jilin University, Changchun, Jilin 130041;
${ }^{2}$ Department of Otolaryngology, Head and Neck Surgery, Tumor Hospital of Jilin Province, Changchun, Jilin 130012, P.R. China
}

Received June 4, 2014; Accepted February 20, 2015

DOI: $10.3892 / \mathrm{mmr} .2015 .3701$

\begin{abstract}
The present study aimed to identify key genes and relevant microRNAs (miRNAs) involved in laryngeal squamous cell carcinoma (LSCC). The gene expression profiles of LSCC tissue samples were analyzed with various bioinformatics tools. A gene expression data set (GSE51985), including ten laryngeal squamous cell carcinoma (LSCC) tissue samples and ten adjacent non-neoplastic tissue samples, was downloaded from the Gene Expression Omnibus. Differential analysis was performed using software package limma of $R$. Functional enrichment analysis was applied to the differentially expressed genes (DEGs) using the Database for Annotation, Visualization and Integrated Discovery. Protein-protein interaction (PPI) networks were constructed for the protein products using information from the Search Tool for the Retrieval of Interacting Genes/Proteins. Module analysis was performed using ClusterONE (a software plugin from Cytoscape). MicroRNAs (miRNAs) regulating the DEGs were predicted using WebGestalt. A total of 461 DEGs were identified in LSCC, 297 of which were upregulated and 164 of which were downregulated. Cell cycle, proteasome and DNA replication were significantly over-represented in the upregulated genes, while the ribosome was significantly over-represented in the downregulated genes. Two PPI networks were constructed for the up- and downregulated genes. One module from the upregulated gene network was associated with protein kinase. Numerous miRNAs associated with LSCC were predicted, including miRNA (miR)-25, miR-32, miR-92 and miR-29. In conclusion, numerous key genes and pathways involved in LSCC were revealed, which may aid the advancement of current knowledge regarding the
\end{abstract}

Correspondence to: Mr. Ying Zheng, Department of Otolaryngology, Head and Neck Surgery, Tumor Hospital of Jilin Province, 1018 Huguang Road, Changchun, Jilin 130012, P.R. China E-mail: yingzhengzz@163.com

Key words: laryngeal squamous cell carcinoma, differentially expressed genes, functional enrichment analysis, protein-protein interaction network, microRNA pathogenesis of LSCC. In addition, relevant miRNAs were also identified, which may represent potential biomarkers for use in the diagnosis or treatment of the disease.

\section{Introduction}

Laryngeal squamous cell carcinoma (LSCC) is the most common type of laryngeal cancer. It is able to spread to regional cervical lymph nodes, or to more distant tissues, for example the lung. Improvements in current therapies have resulted in an improved quality of life for patients with LSCC (1). However, survival rates have not been significantly improved, which identifies the need for a change in clinical approach, requiring novel biomarkers for diagnosis, prognostic assessment and drug design (2).

Certain achievements have been made in the identification of biomarkers of LSCC. Järvinen et al (3) performed high-resolution copy number and gene expression microarray analyses to identify 739 genes overexpressed in LSCC. Gajecka et al (4) reported that polymorphisms of CYP1A1, CYP2D6, CYP2E1, NAT2, GSTM1 and GSTT1 were associated with an increased risk of LSCC. HLA class I antigen downregulation was identified as a poor prognostic marker for LSCC, which may reflect the reduction in the extent of CD8(+) $\mathrm{T}$ cell infiltration in LSCC lesions (5). Overexpression of osteopontin enhances the proliferation and invasiveness of LSCC (6), suggesting that it may represent a potential therapeutic target.

MicroRNAs (miRNAs) are short regulatory RNAs that modulate gene expression at the post-transcriptional level, and are involved in the pathogenesis of numerous types of cancer (7). Multiple miRNAs have been associated with LSCC. Overexpression of miR-21 contributes to the malignant phenotype of LSCC via inhibition of BTG family member 2 (8). miR-203 inhibits the proliferation of laryngeal carcinoma cells by modulating their survival (9). In addition, let-7a (10), miR-16 (11) and hsa-miR-34c (12) also have roles in laryngeal carcinoma.

Microarray technology provides global patterns of gene expression and therefore facilitates biomarker discovery. Lian et al (13) investigated tumorigenesis and regional lymph node metastasis in LSCC, whereas the present study focused specifically on the discovery of biomarkers associated with tumorigenesis. In the present study, gene expression profiles 
of LSCC were analyzed with a variety of bioinformatics tools, including functional enrichment and network analyses, in order to identify novel potential biomarkers. Additionally, miRNAs targeting these genes were also predicted. The identification of such biomarkers may be useful in the diagnosis and/or treatment of LSCC.

\section{Materials and methods}

Gene expression data. A gene expression data set (accession number GSE51985), which included ten LSCC tissue samples and ten adjacent non-neoplastic tissue samples, was downloaded from the Gene Expression Omnibus (http://www.ncbi. nlm.nih.gov/geo) (13). Gene expression levels were measured using the Illumina HumanHT-12 V4.0 expression beadchip platform (Illumina, San Diego, CA, USA; http://www.ncbi. nlm.nih.gov/geo/query/acc.cgi?acc=GPL10558). Platform annotation files were also acquired.

Pretreatment and differential analysis. According to the annotation files, probes were initially mapped into the genes. If more than one probe was mapped into a single gene, levels of the probes were averaged as the final expression level for the specific gene. Following normalization, differential analysis was performed between the ten LSCC tissues and corresponding adjacent non-neoplastic tissues using the Linear Models for Microarray Analysis package (limma; http://www.bioconductor.org/packages/ release/bioc/html/limma.html) (14) of $R . \mathrm{P}<0.05$ and llog2 (fold change) $\mid>1$ were set as the threshold levels for the identification of differentially expressed genes (DEGs).

Functional enrichment analysis. Functional enrichment analysis facilitated the identification of altered biological functions. In the present study, functional enrichment analysis was performed on the DEGs using the Database for Annotation, Visualization and Integration Discovery, version 6.7 (DAVID; http://david.abcc.ncifcrf.gov/) (15), which is able to reveal enriched Gene Ontology (GO) terms; the Kyoto Encyclopedia of Genes and Genomes (KEGG) for pathways (16) and InterPro, version 34.0 for protein domains (17) based on the hypergeometric distribution. $\mathrm{P}<0.05$ was set as the threshold.

Construction of protein-protein interaction (PPI) networks. Proteins 'work together' to exert certain biological functions, and the genome-wide identification of PPIs represents a significant step in the elucidation of the underlying molecular mechanisms. In present study, PPI networks were constructed for the protein products using information from the Search Tool for the Retrieval of Interacting Genes/Proteins (STRING, version 9.1; http://string-db.org/) (18). Interactions with a score (i.e. required confidence) $>0.4$ were retained in the network.

The proteins in the network serve as the 'nodes', and each pairwise protein interaction is represented by an undirected link. The 'degree' of a node corresponds to the number of interactions of that particular protein. The most highly connected nodes (those of a high degree) were considered to be the network 'hubs'.
Module analysis of the network. The PPI networks and regulatory associations between miRNAs and target genes were combined and subsequently visualized with Cytoscape, version 2.6.3 (http://cytoscape.org/) (19). Functional modules of the network were explored using ClusterONE, version 1.0 (http://www.paccanarolab.org/clusterone), a Cytoscape software plugin (20). $\mathrm{P}<0.01$ was set as the cut-off value.

Prediction of miRNAs. The miRNAs regulating the identified DEGs were predicted using WEB-based Gene Set Analysis Toolkit, version 2.0 (WebGestalt; http://bioinfo.vanderbilt. edu/webgestalt/) (21). Count $\geq 2$ and false discovery rate (false positive rate, multiple testing corrected $\mathrm{P}$-value) $<0.1$ were set as the threshold values.

\section{Results}

DEGs in LSCC. According to the criteria outlined (llog2(fold change) $\mid>1$; $\mathrm{P}<0.05$ ), a total of $461 \mathrm{DEGs}$ were identified in LSCC, of which 297 were upregulated and 164 were downregulated.

Functional enrichment analysis. GO and KEGG pathway enrichment analyses were applied to the up- and downregulated genes using DAVID tools. The results are presented in Tables I and II.

The top five gene clusters with enrichment scores $>2$ are listed in Table I. The following molecular functional terms were significantly over-represented amongst the upregulated genes: ATP binding (GO: 0005524), adenyl ribonucleotide binding (GO: 0032559), adenyl nucleotide binding (GO: 0030554), purine nucleoside binding (GO: 0001883), nucleoside binding (GO: 0001882), purine nucleotide binding (GO: 0017076), ribonucleotide binding (GO: 0032553), purine ribonucleotide binding (GO: 0032555) and nucleotide binding (GO: 0000166). Amongst the downregulated genes, structural constituent of ribosome (GO: 0003735) and structural molecule activity (GO: 0005198) were significantly enriched.

In addition, the cell cycle (hsa04110), proteasome (hsa03050) and DNA replication (hsa03030) pathways were significantly enriched in the upregulated genes, while ribosome (hsa03010) was significantly enriched in the downregulated genes.

PPI networks of the DEGs. A PPI network was constructed for the protein products of the DEGs using STRING. Interactions with a combined score of $>0.4$ were included in the network.

A network consisting of 213 proteins (nodes) and 2719 interactions (edges) was generated for the upregulated genes (Fig. 1). The top nodes, or hubs, characterized by a degree of $>70$, were aurora kinase B (AURKB), cyclin dependent kinase (CDK)1, cell division cycle (CDC) 20 homolog, AURKA, CDC45-like, budding uninhibited by benzimidazoles 1 homolog (BUB1), PDZ binding kinase (PBK), non-SMC condensin I complex subunit G (NCAPG), cell division cycle associated 8 (CDCA8), ubiquitin-conjugating enzyme E2T (UBE2T), minichromosome maintenance complex component 2 (MCM2), centromere protein F (CENPF), MCM4, NDC80 homolog kinetochore complex component (NDC80), CENPA, baculoviral IAP repeat-containing 5 (BIRC5), denticleless homolog (DTL), CHK1 checkpoint homolog (CHEK1), kinesin family 
Table I. GO enrichment analysis result for up- and downregulated genes in laryngeal squamous cell carcinoma.

\begin{tabular}{|c|c|c|c|}
\hline Category & Term & Count & P-value \\
\hline \multicolumn{4}{|c|}{ Upregulated genes } \\
\hline Cluster 1 & Enrichment score: 14.33726443940616 & & \\
\hline $\mathrm{BP}$ & GO: 0007049 cell cycle & 63 & $3.98 \times 10^{-27}$ \\
\hline $\mathrm{BP}$ & GO: 0000278 mitotic cell cycle & 46 & $4.67 \times 10^{-27}$ \\
\hline $\mathrm{BP}$ & GO: 0022402 cell cycle process & 50 & $8.03 \times 10^{-23}$ \\
\hline $\mathrm{BP}$ & GO: 0000279 M phase & 37 & $3.88 \times 10^{-20}$ \\
\hline $\mathrm{BP}$ & GO: 0000280 nuclear division & 30 & $1.39 \times 10^{-18}$ \\
\hline $\mathrm{BP}$ & GO: 0007067 mitosis & 30 & $1.39 \times 10^{-18}$ \\
\hline $\mathrm{BP}$ & GO: 0000087 M phase of mitotic cell cycle & 30 & $2.30 \times 10^{-18}$ \\
\hline BP & GO: 0048285 organelle fission & 30 & $4.23 \times 10^{-18}$ \\
\hline $\mathrm{BP}$ & GO: 0022403 cell cycle phase & 38 & $1.02 \times 10^{-17}$ \\
\hline $\mathrm{CC}$ & GO: 0043232 intracellular non-membrane-bounded organelle & 91 & $1.87 \times 10^{-15}$ \\
\hline $\mathrm{CC}$ & GO: 0043228 non-membrane-bounded organelle & 91 & $1.87 \times 10^{-15}$ \\
\hline $\mathrm{BP}$ & GO: 0051301 cell division & 29 & $3.35 \times 10^{-14}$ \\
\hline $\mathrm{CC}$ & GO: 0005819 spindle & 21 & $1.24 \times 10^{-13}$ \\
\hline $\mathrm{CC}$ & GO: 0015630 microtubule cytoskeleton & 35 & $4.61 \times 10^{-12}$ \\
\hline $\mathrm{CC}$ & GO: 0044430 cytoskeletal part & 41 & $6.61 \times 10^{-09}$ \\
\hline $\mathrm{BP}$ & GO: 0007017 microtubule-based process & 20 & $2.68 \times 10^{-08}$ \\
\hline $\mathrm{CC}$ & GO: 0005856 cytoskeleton & 49 & $6.72 \times 10^{-08}$ \\
\hline $\mathrm{CC}$ & GO: 0005815 microtubule organizing center & 17 & $2.28 \times 10^{-06}$ \\
\hline $\mathrm{CC}$ & GO: 0005813 centrosome & 16 & $2.37 \times 10^{-06}$ \\
\hline $\mathrm{CC}$ & GO: 0005874 microtubule & 14 & $3.79 \times 10^{-04}$ \\
\hline Cluster 2 & Enrichment score: 10.562682054877438 & & \\
\hline $\mathrm{CC}$ & GO: 0031981 nuclear lumen & 64 & $1.11 \times 10^{-14}$ \\
\hline $\mathrm{CC}$ & GO: 0070013 intracellular organelle lumen & 69 & $3.57 \times 10^{-13}$ \\
\hline $\mathrm{CC}$ & GO: 0031974 membrane-enclosed lumen & 70 & $8.50 \times 10^{-13}$ \\
\hline $\mathrm{CC}$ & GO: 0043233 organelle lumen & 69 & $1.07 \times 10^{-12}$ \\
\hline $\mathrm{CC}$ & GO: 0005654 nucleoplasm & 38 & $2.84 \times 10^{-08}$ \\
\hline $\mathrm{CC}$ & GO: 0005730 nucleolus & 29 & $4.12 \times 10^{-06}$ \\
\hline Cluster 3 & Enrichment score: 9.848444253761741 & & \\
\hline $\mathrm{CC}$ & GO: 0005694 chromosome & 34 & $1.72 \times 10^{-13}$ \\
\hline $\mathrm{CC}$ & GO: 0000775 chromosome, centromeric region & 19 & $7.04 \times 10^{-13}$ \\
\hline $\mathrm{CC}$ & GO: 0044427 chromosomal part & 30 & $1.90 \times 10^{-12}$ \\
\hline $\mathrm{CC}$ & GO: 0000793 condensed chromosome & 17 & $1.60 \times 10^{-10}$ \\
\hline $\mathrm{CC}$ & GO: 0000779 condensed chromosome, centromeric region & 12 & $5.30 \times 10^{-09}$ \\
\hline $\mathrm{CC}$ & GO: 0000777 condensed chromosome kinetochore & 11 & $1.91 \times 10^{-08}$ \\
\hline $\mathrm{CC}$ & GO: 0000776 kinetochore & 11 & $3.10 \times 10^{-07}$ \\
\hline Cluster 4 & Enrichment score: 7.286973695097799 & & \\
\hline $\mathrm{MF}$ & GO: 0005524 adenosine triphosphate binding & 52 & $9.82 \times 10^{-09}$ \\
\hline MF & GO: 0032559 adenyl ribonucleotide binding & 52 & $1.54 \times 10^{-08}$ \\
\hline MF & GO: 0030554 adenyl nucleotide binding & 53 & $3.18 \times 10^{-08}$ \\
\hline MF & GO: 0001883 purine nucleoside binding & 53 & $5.25 \times 10^{-08}$ \\
\hline MF & GO: 0001882 nucleoside binding & 53 & $6.58 \times 10^{-08}$ \\
\hline MF & GO: 0017076 purine nucleotide binding & 59 & $8.65 \times 10^{-08}$ \\
\hline MF & GO: 0032553 ribonucleotide binding & 57 & $1.19 \times 10^{-07}$ \\
\hline MF & GO: 0032555 purine ribonucleotide binding & 57 & $1.19 \times 10^{-07}$ \\
\hline MF & GO: 0000166 nucleotide binding & 65 & $1.29 \times 10^{-07}$ \\
\hline Cluster 5 & Enrichment score: 5.683294675111861 & & \\
\hline $\mathrm{BP}$ & GO: 0007017 microtubule-based process & 20 & $2.68 \times 10^{-08}$ \\
\hline $\mathrm{BP}$ & GO: 0000226 microtubule cytoskeleton organization & 15 & $1.06 \times 10^{-07}$ \\
\hline $\mathrm{BP}$ & GO: 0007051 spindle organization & 7 & $7.88 \times 10^{-05}$ \\
\hline $\mathrm{BP}$ & GO: 0007010 cytoskeleton organization & 20 & $8.29 \times 10^{-05}$ \\
\hline
\end{tabular}


Table I. Continued.

\begin{tabular}{|c|c|c|c|}
\hline Category & Term & Count & P-value \\
\hline \multicolumn{4}{|c|}{ Downregulated genes } \\
\hline Cluster 1 & Enrichment score: 2.991494823613603 & & \\
\hline $\mathrm{BP}$ & GO: 0006414 translational elongation & 9 & $3.72 \times 10^{-07}$ \\
\hline MF & GO: $0003735 \sim$ structural constituent of ribosome & 10 & $2.59 \times 10^{-06}$ \\
\hline $\mathrm{CC}$ & GO: 0033279 ribosomal subunit & 9 & $4.39 \times 10^{-06}$ \\
\hline $\mathrm{CC}$ & GO: 0022626 cytosolic ribosome & 7 & $2.80 \times 10^{-05}$ \\
\hline $\mathrm{CC}$ & GO: 0005840 ribosome & 10 & $2.81 \times 10^{-05}$ \\
\hline $\mathrm{BP}$ & GO: 0006412 translation & 11 & $7.45 \times 10^{-05}$ \\
\hline $\mathrm{CC}$ & GO: 0015934 large ribosomal subunit & 6 & $1.30 \times 10^{-04}$ \\
\hline $\mathrm{CC}$ & GO: 0044445 cytosolic part & 7 & $8.82 \times 10^{-04}$ \\
\hline $\mathrm{CC}$ & GO: 0022625 cytosolic large ribosomal subunit & 4 & $2.70 \times 10^{-03}$ \\
\hline $\mathrm{CC}$ & GO: 0030529 ribonucleoprotein complex & 11 & $4.49 \times 10^{-03}$ \\
\hline MF & GO: $0005198 \sim$ structural molecule activity & 12 & $4.68 \times 10^{-03}$ \\
\hline
\end{tabular}

Cluster, functional cluster; enrichment score, score for a functional cluster reflecting clustering effect; BP, biological process; CC, cellular components; MF, molecular function; count, number differentially expressed in a specific pathway; GO, gene ontology.

Table II. Kyoto Encyclopedia of Genes and Genomes pathway enrichment analysis result for up-regulated genes in laryngeal squamous cell carcinoma.

\begin{tabular}{|c|c|c|}
\hline Term & Count & P-value \\
\hline \multicolumn{3}{|l|}{ Upregulated genes } \\
\hline Hsa04110: Cell cycle & 16 & $4.12 \times 10^{-09}$ \\
\hline Hsa03050: Proteasome & 11 & $6.84 \times 10^{-09}$ \\
\hline Hsa03030: DNA replication & 6 & $4.21 \times 10^{-04}$ \\
\hline Hsa04114: Oocyte meiosis & 8 & $3.54 \times 10^{-03}$ \\
\hline Hsa03040: Spliceosome & 8 & $7.43 \times 10^{-03}$ \\
\hline \multicolumn{3}{|l|}{ Downregulated genes } \\
\hline Hsa03010: Ribosome & 8 & $5.59 \times 10^{-06}$ \\
\hline Hsa00071: Fatty acid metabolism & 4 & $4.19 \times 10^{-03}$ \\
\hline
\end{tabular}

member 2C (KIF2C), maternal embryonic leucine zipper kinase (MELK), topoisomerase (DNA) II $\alpha$ 170kDa (TOP2A) and cell division cycle associated 5 (CDCA5). Furthermore, a network comprised of 50 nodes and 80 edges was obtained for the downregulated genes. Few PPIs were observed amongst the downregulated genes, and therefore the subsequent analyses were focused on the upregulated genes.

Module analysis. Module analysis was performed using ClusterONE to predict protein complexes, and the results are presented in Fig. 2. Two modules were identified amongst the upregulated genes: Module $1(\mathrm{P}<0.000)$ and Module 2 $\left(\mathrm{P}=5.559 \times 10^{-4}\right)$. Protein domain enrichment analysis was applied to the genes in the two modules using InterPro and the results are displayed in Table III. Serine/threonine protein kinase, active site (IPR008271); protein kinase, ATP binding site (IPR017441) and protein kinase, core (IPR000719) were significantly enriched amongst the genes from Module 1. No significantly enriched term was identified in the genes from Module 2. One module was generated by the downregulated genes (Fig. 2), but no significantly enriched protein domain was identified.

miRNA-target interactions. miRNAs regulating the DEGs were predicted by WebGestalt, and the results are exhibited in Table IV and Fig. 3. hsa_GTGCAAT, miR-25, miR-32, miR-92, miR-363, miR-367, hsa_TGCTGCT, miR-15A, miR-16, miR-15B, miR-195, miR-424, miR-497, hsa_TGGTGCT, miR-29A, miR-29B and miR-29C were included in the list of identified miRNAs.

Gene regulatory networks. The miRNA-target interactions were visualized by Cytoscape and thereby a gene regulatory network was established (Fig. 3).

\section{Discussion}

Through the comparative analysis of gene expression data of LSCC and adjacent non-neoplastic control tissues, a total of 461 DEGs were identified in LSCC, of which 297 were 
Table III. Significantly over-represented protein domains in the genes from the Module 1 network of upregulated genes.

\begin{tabular}{lcc}
\hline Term & Count & P-value \\
\hline IPR008271: Serine/threonine protein kinase, active site & 11 & $1.61 \times 10^{-06}$ \\
IPR017441: Protein kinase, ATP binding site & 12 & $2.11 \times 10^{-06}$ \\
IPR000719: Protein kinase, core & 12 & $3.25 \times 10^{-06}$ \\
IPR002290: Serine/threonine protein kinase & 9 & $1.01 \times 10^{-05}$ \\
IPR017442: Serine/threonine protein kinase-related & 10 & $1.46 \times 10^{-05}$ \\
IPR018525: DNA-dependent ATPase MCM, conserved site & 3 & $4.52 \times 10^{-04}$ \\
IPR001208: DNA-dependent ATPase MCM & 3 & $5.80 \times 10^{-04}$ \\
IPR019821: Kinesin, motor region, conserved site & 4 & $6.66 \times 10^{-04}$ \\
IPR001752: Kinesin, motor region & 4 & $6.66 \times 10^{-04}$ \\
IPR007125: Histone core & 4 & $8.16 \times 10^{-04}$ \\
IPR009072: Histone-fold & 4 & $1.39 \times 10^{-03}$ \\
IPR002119: Histone H2A & 3 & $2.68 \times 10^{-03}$ \\
\hline
\end{tabular}

ATP, adenosine triphosphate; MCM, minichromosome maintenance complex component.

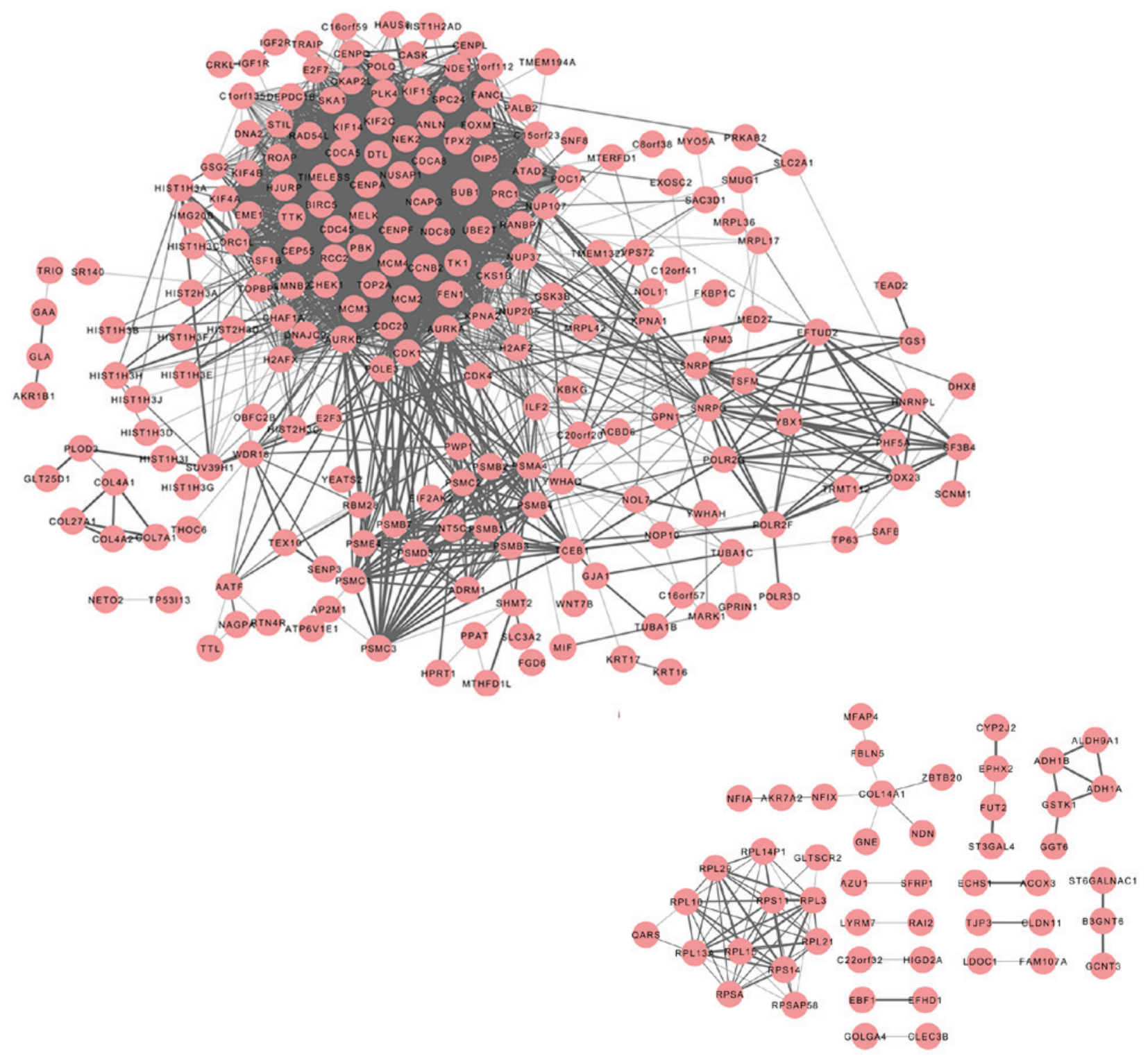

Figure 1. Protein-protein interaction networks for (A) upregulated genes and (B) downregulated genes in laryngeal squamous cell carcinoma. Red circles, protein products of differentially expressed genes; black lines, interactions between proteins. The thickness of the line is positively correlated with the degree of the interaction. 
Table IV. Predicted miRNAs regulating the DEGs.

\begin{tabular}{lccccccr}
\hline miRNA & Count & C & O & E & R & rawP & adjP \\
\hline $\begin{array}{l}\text { Hsa_GTGCAAT, miR-25, miR-32, miR-92, } \\
\text { miR-363, miR-367 }\end{array}$ & 12 & 308 & 12 & 3.42 & 3.51 & 0.0002 & 0.0123 \\
Hsa_TGCTGCT, miR-15A, miR-16, miR-15B, & 18 & 593 & 18 & 6.59 & 2.73 & 0.0001 & 0.0123 \\
miR-195, miR-424, miR-497 & & & & & & & \\
Hsa_TGGTGCT, miR-29A, miR-29B, miR-29C & 15 & 515 & 15 & 5.72 & 2.62 & 0.0007 & 0.0287 \\
Hsa_ACTACCT, miR-196A, miR-196B & 7 & 143 & 7 & 1.59 & 4.41 & 0.0011 & 0.0338 \\
Hsa_ATATGCA, miR-448 & 8 & 208 & 8 & 2.31 & 3.46 & 0.0024 & 0.0492 \\
Hsa_TAATGTG, miR-323 & 7 & 158 & 7 & 1.75 & 3.99 & 0.0020 & 0.0492 \\
Hsa_AACTGAC, miR-223 & 5 & 94 & 5 & 1.04 & 4.79 & 0.0040 & 0.0703 \\
Hsa_TATCTGG, miR-488 & 4 & 62 & 4 & 0.69 & 5.81 & 0.0050 & 0.0724 \\
Hsa_TGTTTAC, miR-30A-5P, miR-30C, & 14 & 572 & 14 & 6.35 & 2.20 & 0.0053 & 0.0724 \\
miR-30D, miR-30B, miR-30E-5P & & & & & & & \\
Hsa_CAGCAGG, miR-370 & 6 & 153 & 6 & 1.70 & 3.53 & 0.0075 & 0.0922 \\
\hline
\end{tabular}

miRNA, micro RNA; DEGs, differentially expressed genes; miR, micro RNA; Count, number of DEGS regulated by a specific miRNA; C, number of reference genes in the category; $\mathrm{O}$, number of genes in the gene set and also in the category; E, expected number in the category; R, ratio of enrichment; rawP, raw P-value calculated by WebGastalt; adjP, adjusted P-value.
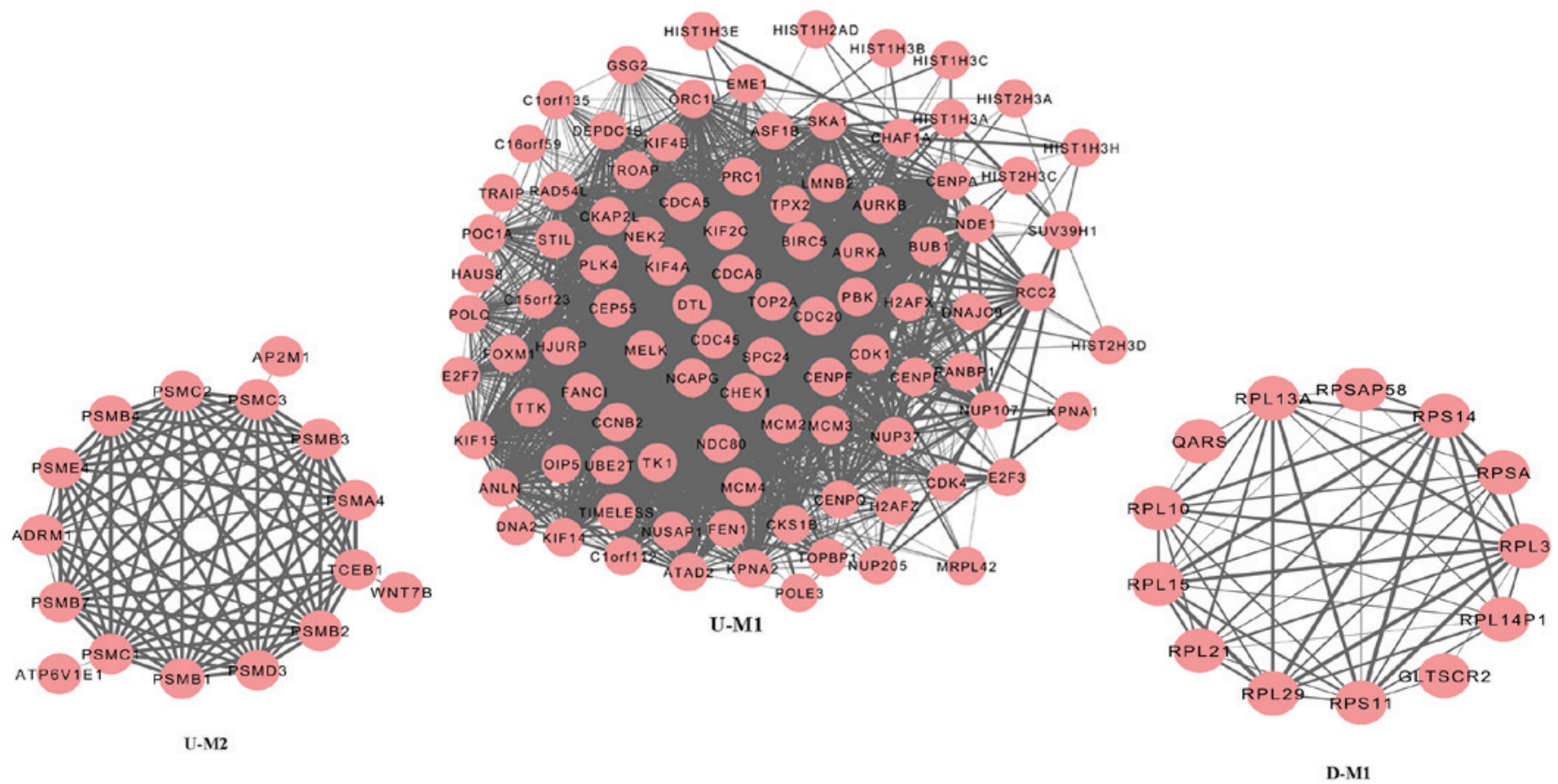

Figure 2. Modules identified from the protein-protein interaction networks. U-M1, Module 1 from the network of upregulated genes. U-M2, Module 2 from the network of upregulated genes. D-M1, Module 1 from the network of downregulated genes.

upregulated and 164 were downregulated. Functional enrichment analysis indicated that the cell cycle, proteasome and relevant biological pathways were over-represented amongst the upregulated genes. Cell cycle progression is closely associated with multiple types of cancer (22-23), which indicated that the analysis results were of high confidence.

According to the results of previous studies, specific DEGs including CDK4, CDK1, MCM2, MCM3 and MCM4, have been implicated in LSCC $(13,24)$. CDK4 is required for cell cycle $G_{1}$ phase progression (25). Dong et al (26) reported that CDK4 was overexpressed in LSCC and suggested that it may have a critical role in cell proliferation together with cyclin D1. MCM2 has been implicated in the initiation of eukaryotic genome replication, which has been proposed as a marker of dysplasia and malignancy (27). Chatrath et al (28) reported aberrant expression of MCM2 in LSCC, while Torres-Rendon et al (29) indicated that MCM2 may be an indicator of growth and may provide a useful prognostic tool for oral epithelial dysplasia. MCM3 and MCM4 were also identified as DEGs in the present study. These findings were consistent with the results presented in a study by Lian et al (13). 


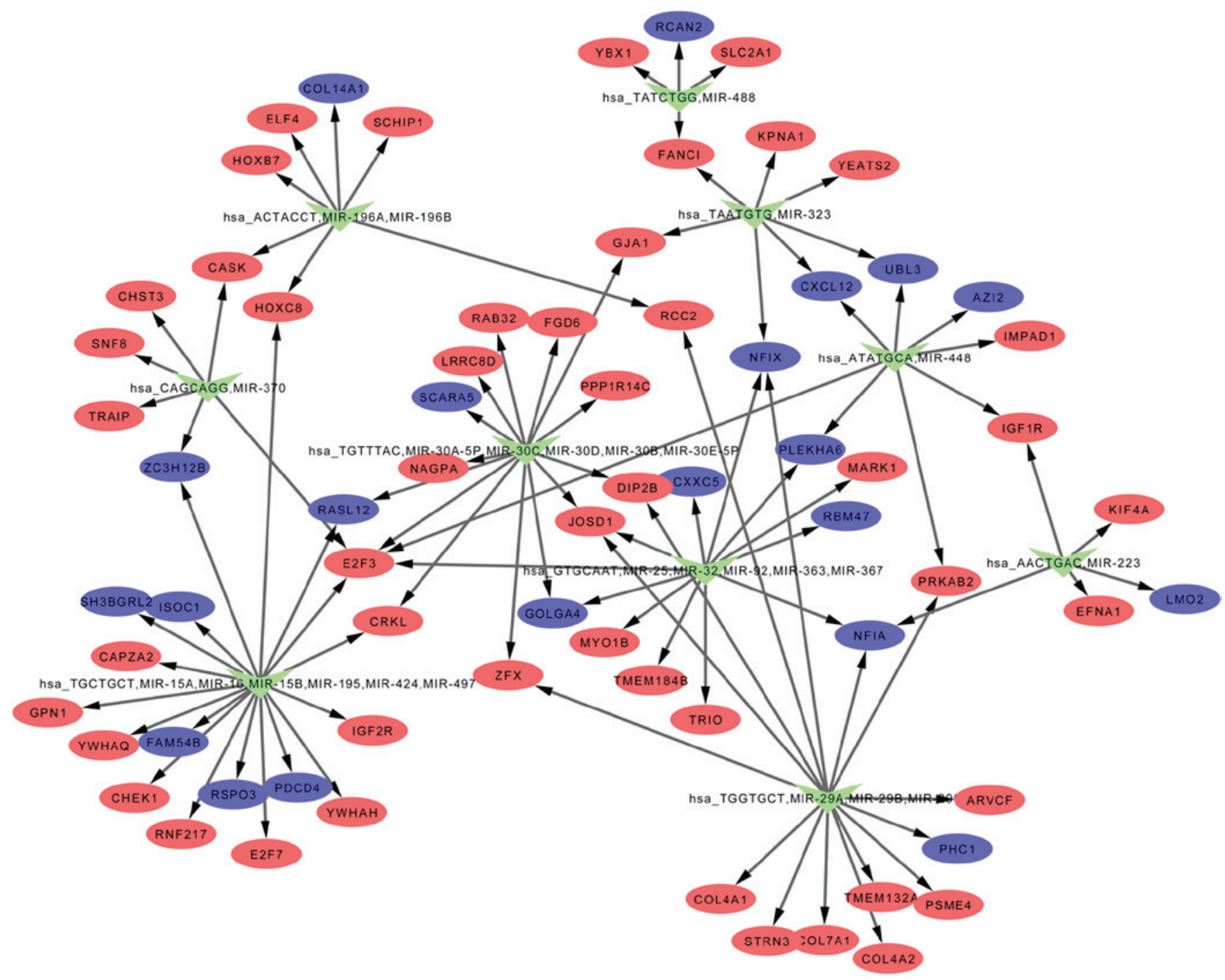

Figure 3. Gene regulatory network for the differentially expressed genes. Green triangles, micro RNAs; red ellipses, upregulated genes and blue ellipses, downregulated genes. The regulatory associations are represented by arrow lines.

Additional potential biomarkers were discovered in the present study. There is emerging evidence that glycogen synthase kinase (GSK) $3 \beta$ may be a tumor suppressor in oral cancer (30). It was therefore hypothesized that GSK3 $\beta$ may function in a similar way in LSCC. Several subunits of the proteasome were also identified in LSCC, including proteasome subunit $\beta$ type 4 (PSMB4), PSMB7, PSMB1 and PSMC3. The ubiquitin-proteasome system is a critical regulator of cell growth and apoptosis (31), and proteasome inhibitors have been developed for use in cancer therapy (32-33). PSMB4 was identified as the first proteasomal subunit with oncogenic properties, promoting cancer cell survival and tumor growth in vivo (34). Elevated expression of PSMB4 is associated with poor prognosis in human cancer (34). PSMB7 was identified to be a prognostic biomarker in breast cancer (35). Therefore, further study of these subunits may reveal novel biomarkers for LSCC.

Network and module analyses were performed for the DEGs, and the identification of hub nodes and interactions may aid the elucidation of the underlying molecular mechanisms. AURKA and AURKB had a high degree in the PPI network for upregulated genes. Overexpression and hyperactivation of AURKA and AURKB have major roles in tumorigenesis, and therefore their inhibitors are already regarded as promising therapeutics for various types of cancer (36), including head and neck squamous-cell carcinoma (37). MCM2 and MCM4 also had a degree of $>70$, confirming their significant roles in the pathogenesis of LSCC.

Considering that miRNAs are closely involved in multiple types of cancer, a number of miRNAs targeting the DEGs were predicted by WebGestalt, including miR-15, miR-16, miR-25 and miR-195. Wu et al (11) reported that miR-16 was upregulated in LSCC and that it targets zyxin and promotes cell motility in human laryngeal carcinoma cell line HEp-2. Other miRNAs have been reported to have roles in various types of cancer. miR-15a forms a cluster with miR-16 at the chromosomal region $13 q 14$, and functions as a putative tumor suppressor by targeting the oncogene BCL2 $(38,39)$. miR-25 regulates apoptosis by targeting Bim in human ovarian cancer (40) and miR-195 is regarded as a predictor of poor prognosis in adrenocortical cancer (41). Future studies of these miRNAs may better describe the regulatory mechanisms underlying LSCC. 
In conclusion, a number of key genes in LSCC were identified, which may represent novel biomarkers for diagnosis, prognosis and therapy. In addition, relevant miRNAs were also explored, and these may offer therapeutic targets for the modulation of abnormal gene expression in LSCC.

\section{References}

1. Schorn VJ and Miles BA: Laryngeal squamous cell carcinoma. In: ENT Board Prep. Lin F and Patel Z (eds). Springer, New York, pp227-233, 2014.

2. Almadori G, Bussu F, Cadoni G, Galli J, Paludetti G and Maurizi M: Molecular markers in laryngeal squamous cell carcinoma: towards an integrated clinicobiological approach. Eur J Cancer 41: 683-693, 2005.

3. Järvinen AK, Autio R, Haapa-Paananen S, et al: Identification of target genes in laryngeal squamous cell carcinoma by high-resolution copy number and gene expression microarray analyses. Oncogene 25: 6997-7008, 2006.

4. Gajecka M, Rydzanicz M, Jaskula-Sztul R, Kujawski M, Szyfter W and Szyfter K: CYP1A1, CYP2D6, CYP2E1, NAT2, GSTM1 and GSTT1 polymorphisms or their combinations are associated with the increased risk of the laryngeal squamous cell carcinoma. Mutat Res 574: 112-123, 2005

5. Ogino T, Shigyo H, Ishii $\mathrm{H}$, et al: HLA class I antigen down-regulation in primary laryngeal squamous cell carcinoma lesions as a poor prognostic marker. Cancer Res 66: 9281-9289, 2006.

6. Celetti A, Testa D, Staibano S, et al: Overexpression of the cytokine osteopontin identifies aggressive laryngeal squamous cell carcinomas and enhances carcinoma cell proliferation and invasiveness. Clin Cancer Res 11: 8019-8027, 2005.

7. Zimmerman AL and Wu S: MicroRNAs, cancer and cancer stem cells. Cancer Lett 300: 10-19, 2011.

8. Liu M, Wu H, Liu T, et al: Regulation of the cell cycle gene, BTG2, by miR-21 in human laryngeal carcinoma. Cell Res 19: 828-837, 2009

9. Bian K, Fan J, Zhang X, et al: MicroRNA-203 leads to G1 phase cell cycle arrest in laryngeal carcinoma cells by directly targeting survivin. FEBS Lett 586: 804-809, 2012.

10. Long XB, Sun GB, Hu S, et al: Let-7a microRNA functions as a potential tumor suppressor in human laryngeal cancer. Oncol Rep 22: 1189-1195, 2009.

11. Wu H, Liu T, Wang R, et al: MicroRNA-16 targets zyxin and promotes cell motility in human laryngeal carcinoma cell line HEp-2. IUBMB Life 63: 101-108, 2011.

12. Cai KM, Bao XL, Kong XH, et al: Hsa-miR-34c suppresses growth and invasion of human laryngeal carcinoma cells via targeting c-Met. Int J Mol Med 25: 565-571, 2010.

13. Lian M, Fang J, Han D, et al: Microarray gene expression analysis of tumorigenesis and regional lymph node metastasis in laryngeal squamous cell carcinoma. PLoS One 8: e84854, 2013.

14. Diboun I, Wernisch L, Orengo CA and Koltzenburg M: Microarray analysis after RNA amplification can detect pronounced differences in gene expression using limma. BMC Genomics 7: 252, 2006

15. Huang DW, Sherman BT, Tan Q, et al: The DAVID gene functional classification tool: a novel biological module-centric algorithm to functionally analyze large gene lists. Genome Biol 8: R183, 2007.

16. Aoki-Kinoshita KF and Kanehisa M: Gene annotation and pathway mapping in KEGG. Methods Mol Biol 396: 71-91, 2007.

17. Hunter S, Jones P, Mitchell A, et al: InterPro in 2011: new developments in the family and domain prediction database. Nucleic Acids Res 40: D306-D312, 2012.
18. von Mering C, Huynen M, Jaeggi D, Schmidt S, Bork P and Snel B: STRING: a database of predicted functional associations between proteins. Nucleic Acids Res 31: 258-261, 2003.

19. Shannon P, Markiel A, Ozier O, et al: Cytoscape: a software environment for integrated models of biomolecular interaction networks. Genome Res 13: 2498-2504, 2003.

20. Nepusz T, Yu H and Paccanaro A: Detecting overlapping protein complexes in protein-protein interaction networks. Nat Methods 9: 471-472, 2012.

21. Zhang B, Kirov S and Snoddy J: WebGestalt: an integrated system for exploring gene sets in various biological contexts. Nucleic Acids Res 33: W741-W748, 2005.

22. Evan GI and Vousden KH: Proliferation, cell cycle and apoptosis in cancer. Nature 411: 342-348, 2001.

23. Kastan MB and Bartek J: Cell-cycle checkpoints and cancer. Nature 432: 316-323, 2004.

24. Cai K, Luo Y and Li L: Expression of MCM2 protein and its biological characteristic in human laryngeal squamous cell carcinoma. J Acta Academiae Medicinae Jiangxi 3: 58-60, 2008.

25. Sherr CJ: G1 phase progression: cycling on cue. J Cell 79: 551-555, 1994

26. Dong Y, Sui L, Sugimoto K, Tai Y and Tokuda M: Cyclin D1. CDK4 complex, a possible critical factor for cell proliferation and prognosis in laryngeal squamous cell carcinomas. Int $\mathrm{J}$ Cancer 95: 209-215, 2001.

27. Freeman A, Morris LS, Mills AD, et al: Minichromosome maintenance proteins as biological markers of dysplasia and malignancy. Clin Cancer Res 5: 2121-2132, 1999.

28. Chatrath P, Scott IS, Morris LS, et al: Aberrant expression of minichromosome maintenance protein-2 and Ki67 in laryngeal squamous epithelial lesions. Br J Cancer 89: 1048-1054, 2003.

29. Torres-Rendon A, Roy S, Craig G and Speight P: Expression of $\mathrm{Mcm} 2$, geminin and Ki67 in normal oral mucosa, oral epithelial dysplasias and their corresponding squamous-cell carcinomas. Br J Cancer 100: 1128-1134, 2009.

30. Mishra R: Glycogen synthase kinase 3 beta: can it be a target for oral cancer. Mol Cancer 9: 144, 2010.

31. Almond JB and Cohen GM: The proteasome: a novel target for cancer chemotherapy. Leukemia 16: 433-443, 2002.

32. Orlowski RZ and Kuhn DJ: Proteasome inhibitors in cancer therapy: lessons from the first decade. Clin Cancer Res 14: 1649-1657, 2008

33. Rajkumar SV, Richardson PG, Hideshima T and Anderson KC: Proteasome inhibition as a novel therapeutic target in human cancer. J Clin Oncol 23: 630-639, 2005.

34. Lee GY, Haverty PM, Li L et al: Comparative oncogenomics identifies PSMB4 and SHMT2 as potential cancer driver genes. Cancer Res 74: 3114-3126, 2014.

35. Munkacsy G, Abdul-Ghani R, Mihaly Z, et al: PSMB7 is associated with anthracycline resistance and is a prognostic biomarker in breast cancer. Br J Cancer 102: 361-368, 2010.

36. Dar AA, Goff LW, Majid S, Berlin J and El-Rifai W: Aurora kinase inhibitors-rising stars in cancer therapeutics? Mol Cancer Ther 9: 268-278, 2010.

37. Mehra R, Serebriiskii IG, Burtness B, Astsaturov I and Golemis EA: Aurora kinases in head and neck cancer. Lancet Oncol 14: e425-e435, 2013.

38. Bonci D, Coppola V, Musumeci M, et al: The miR-15a-miR-16-1 cluster controls prostate cancer by targeting multiple oncogenic activities. Nat Med 14: 1271-1277, 2008

39. Cimmino A, Calin GA, Fabbri M, et al: miR-15 and miR-16 induce apoptosis by targeting BCL2. Proc Natl Acad Sci USA 102: 13944-13949, 2005.

40. Zhang H, Zuo Z, Lu X, Wang L, Wang H and Zhu Z: MiR-25 regulates apoptosis by targeting Bim in human ovarian cancer. Oncol Rep 27: 594-598, 2012

41. Soon PS, Tacon LJ, Gill AJ, et al: miR-195 and miR-483-5p identified as predictors of poor prognosis in adrenocortical cancer. Clin Cancer Res 15: 7684-7692, 2009. 